www.nature.com/jp

\title{
ERRATUM \\ Response to Darmstadt et al.
}

Journal of Perinatology (2007) 27, 531; doi:10.1038/sj.jp.7211765

Correction to: Journal of Perinatology (2007) 27, 255.

doi:10.1038/sj.jp.7211670
Following the publication of this article, the editor discovered that the author's name was incorrect; the correct name is shown above. 